\title{
2. Introduction to Computers (2)
}

A computer system generally consists of:

1. the computer itself (also called the Central Processing Unit) - an electronic device which is able to follow a set of instructions given it in advance telling it how to do a particular job;

and

2. a number of additional devices (called peripherals) which are connected to the computer and provide:

a) a way of giving the computer information it will need - such as a keyboard allowing it to be typed in; (these pieces of equipment are known as input devices);

b) a way of allowing the computer to give information to the user such as a screen or a printer; (output devices);

and

c) a way of allowing the computer to store information long-term such as a disk-drive unit; (backing-storage devices).

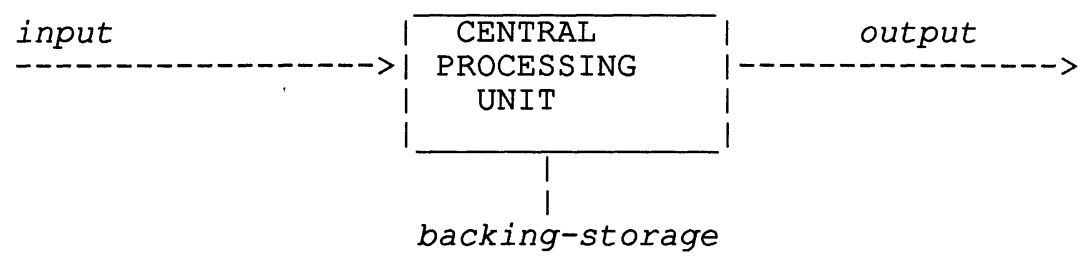

The Central Processing Unit itself consists of:

1. the Control Unit - which controls the rest of the computer;

2. the Arithmetic and Logic Unit - which as its name implies carries out calculations and comparisons;

3. the Central Memory - which is used as temporary storage for instructions and information the computer is using at the time;

4. a number of other devices to help the computer communicate with peripherals and work efficiently.

A computer will not operate without sets of instructions telling it what to do. These fall into two categories:

1. instructions to carry out basic functions such as looking after filing systems on the disk, communication with the printer and handling commands from users; this set of instructions is called the operating system and it has to be loaded into the computer as soon as it is switched on - otherwise the machine will not be able to work efficiently;

2. instructions to carry out particular jobs for the user - such as word processing, accounting or payroll; these are known as applications programs and can be loaded into the computer at any time they are needed (once the operating system has been loaded). 